\title{
The Development of a Modular Platform for Convergence Service in Home Network
}

\author{
M. Humayun Kabir ${ }^{1}$, M. Robiul Hoque ${ }^{1}$, Kangji Lee ${ }^{2}$, Chang-Won Park ${ }^{3}$, \\ and Sung-Hyun Yang ${ }^{*}$ \\ ${ }^{1}$ Department of Electronic Engineering, Kwangwoon University \\ Seoul, Republic of Korea \\ ${ }^{2}$ MIware Inc., Gyeonggi-do, Republic of Korea \\ ${ }^{3}$ Korea Electronics Technology Institution (KETI), Republic of Korea \\ shyang@kw.ac.kr
}

\begin{abstract}
Home network service area increases and different types of service hardware are used for each service, as a result complexity also increases. It is necessary to develop a convergence service platform for home network. In this paper, a modular type home network service platform is proposed and implemented for different types of services. This platform is equipped with sufficient interface and expansion facilities for different service module. It uses Real Time Operating System (RTOS), framework and Platform Application Interface (API) for services. It has flexibility to supporteach service depending on the selected hardware module. It provides convergence service (Home device control and status monitoring management service, healthcare service, community computing service, etc) platform for home user.
\end{abstract}

Keywords: Modular type platform, Convergence service, Real Time embedded system, Home network

\section{Introduction}

A home network is an integration of three kinds of networks: the information network, the multimedia network and the home appliances network [1]. A home network can share data and information among devices attached in the network. At present different types of wired (Ethernet, USB, PLC, etc.,) and wireless (Zigbee, Wi-Fi, Bluetooth, etc.,) home networking technologies have been developed [2]. For the ease of management a variety of device access protocols (HAVi, UPnP, Jini, LonWorks, CEBus, X10, etc.,) also have been proposed. There are four major areas of public demand for home networking services using networked devices [3]. Home automation systems have been steadily gaining popularity especially in homes and office spaces. These systems are installed with the intention of providing the user with easier access and more control over the devices in the home. S. Guangming et al., present a multiinterface sensor network gateway architecture for home automation and other distributed monitoring application scenarios in home environments, ranging from on-site configuration to mobile access [4]. The user will be able to monitor and control devices at home locally as well as remotely via the internet provided the users has access to a device with a compatible web browser. Generally, home automation systems can be implemented within any physical structure provided the limitations of the technology being used are taken into account prior to

* Corresponding Author 
installation. A complete home automation system consists of a Wi-Fi router or an internet connection, a smart home gateway and multiple nodes (known as end devices). Advanced systems may also incorporate cutting edge devices such as digital picture frames and interactive touch surfaces. Appliances and other devices around the home are networked together with the help of a home gateway, which acts as the brain of the system. The numerous technologies presently available for home automation systems offer various advantages and disadvantages. These include the range of which devices can be placed relative to the home gateway, access delays, problems with interference and the security of the communication protocols [5].

The percentage of elderly populations in industrialized nations who are affected by disabilities and chronic diseases, is increasing. In recent years novel care models based on home networking have been proposed [6]. Through those systems, care centers and hospitals are capable of continuously collecting the biomedical signals of those being cared for and those individuals can also enjoy on-line services for clinic appointments, medical consultation, remote alarming, etc.

High quality television broadcasts are transmitted over satellite, able or broadband internet connections. IPTV is a relatively new and emerging alternative service that transmits TV signals over broadband internet. In IPTV service, operators install a network switch at the customer's broadband connection, and install Ethernet cables from the switch to television sets [7].

Conventionally, dedicated hardware (Set-topbox, Gateway Modem, etc.,) with network is used for each service. This approach faces several drawbacks: Devices in different networks cannot share resources, cannot collaborate service need to be developed in different platforms and cannot inter-operate. To solved these drawbacks it is necessary to integrate all the home networks into one platformand allow for further development and expansion. This is a modular type service platform and it will provide convergence service. Modular type platform fulfills various functions through a combination of distinct buildings blocks (Modules) [8]. Building a modular type service platform for convergence services with expansion facilities could reduce the complexity and performance cost ratio of future development. Our aim is to develop a low power modular type integrated platform with 32bit processor, differentrypes of interacing facilities and open source real time operating system. This is called a home service platform. It is similar to a digital device hub for home network as shown in Figure 1 Thi platform can support a modular type open home network framework and provide user selectable services. Different platform APPs are developed for different kind of services.

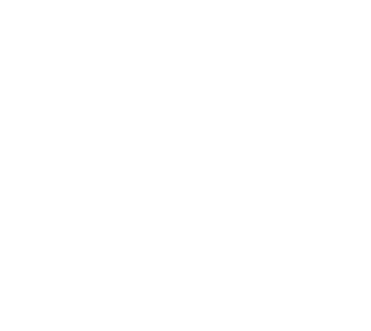




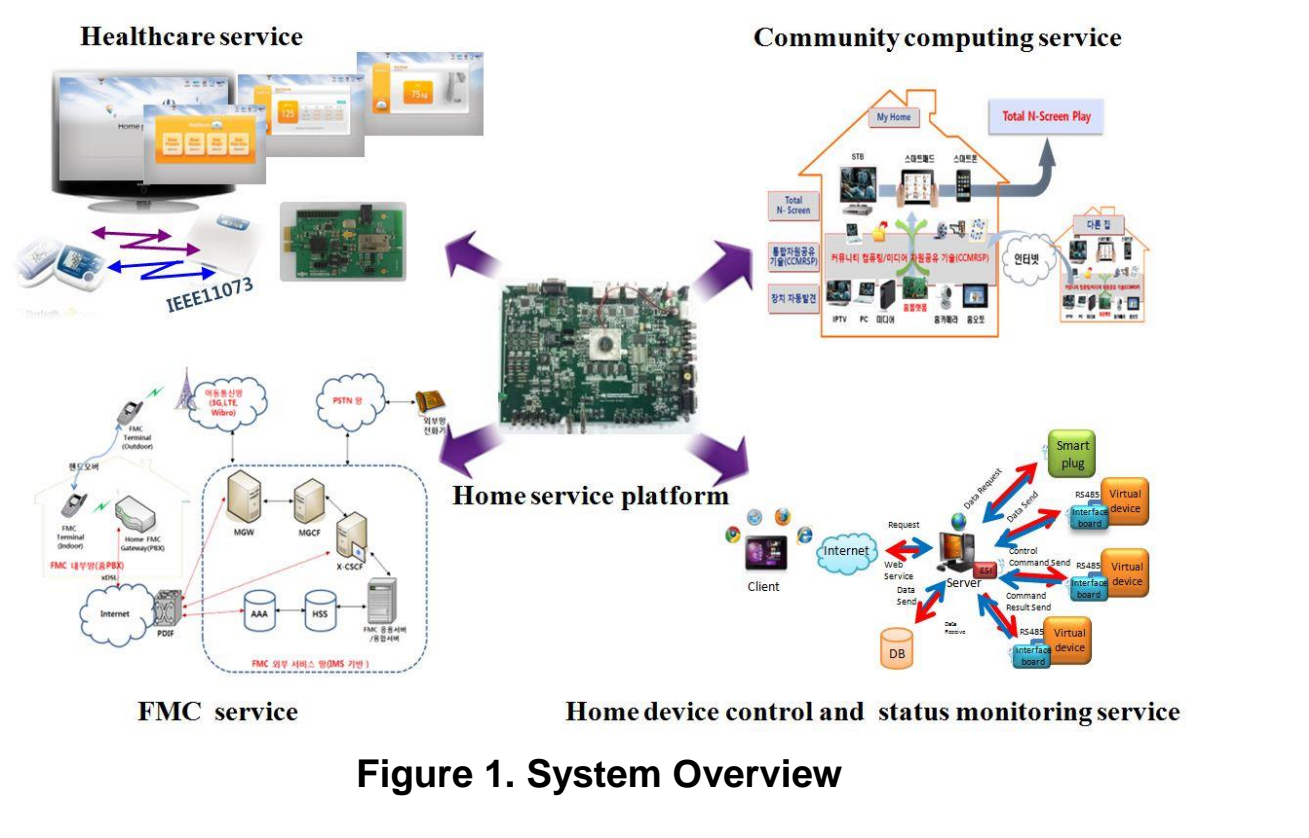

The rest of the paper is organized as follows. In Section 2, we discuss the details of our developed home service platform. Section 3 shaws the implenientation results followed by an overall conclusion in Section 4.

\section{The Design of Home Service Platiorm}

In this section the overall design architecture of the home service platform is presented. It is divided into several sections: The main processor section, the peripheral interface section, the network interface section, the system inferface section and the power section.

\subsection{The Main Processor Section}

A 32-bit high-performance processor is used in the developed home service platform. This processor supports embedded operating system and peripheral integrations ideally suitable for communication and netwôrk pröcessing applications including radio, gateways, switches and network attached storage. It is also useful for single board computers and distributed systems as well as human machinc interfaces and operator panels.

\subsection{The Peripheral Section}

The developed home service platform has different peripheral interface ports to control external peripheral devices and communicate with external processors as shown in Figure 2. Different types of peripherals are used for serial interfaces, program/data storage, DMA, system control and connectivity. The HD Video Processing Subsystem (HDVPSS) provides the output of simultaneous HD and SD analog video and dual HD video inputs. The connectivity peripheral set includes two USB ports with integrated 2.0 PHY, PCIe port x2 lanes and a GEN2 compliant interface, which allows the device to act as a PCIe root complex or device endpoint. A switching device is used for multiport PCIe ports. The serial peripheral set includes, one 6-channel McASP audio serial port (with DIT mode), two dual-channel McASP audio serial ports (with DIT mode), one McBSP multichannel buffered serial port, three UARTs with IrDA and CIR support, SPI serial interface, two I2C master/slave interfaces. The program/data storage peripheral set includes, a SD/SDIO serial interface, dual 
DDR2 SDRAM interface, flexible 8/16-bit asynchronous memory interface, and two SATA interfaces for external storage on two disk drives, or even more with the use of a port multiplier. The system control peripheral set includes seven 32-bit general purpose timers, a real-time clock and a system watchdog timer. On board connectors allows ease of interfacing to the daughter boards. Health care module uses PCIe (as shown in Figure 2).

\subsection{The Network Interface Section}

This section composes of Ethernet transceiver module, Gigabit Ethernet transceiver module and WLAN module (Wi-Fi). Ethernet transceiver module is interface by GPMC, Gigabit Ethernet (10/100/1000 Mbps) transceiver by GMII and WLAN by SD/SDIO. Home device control and status monitoring management service module uses WiFi, and Community computing service uses GMII for connecting with the host processor (as shown in Figure 2).

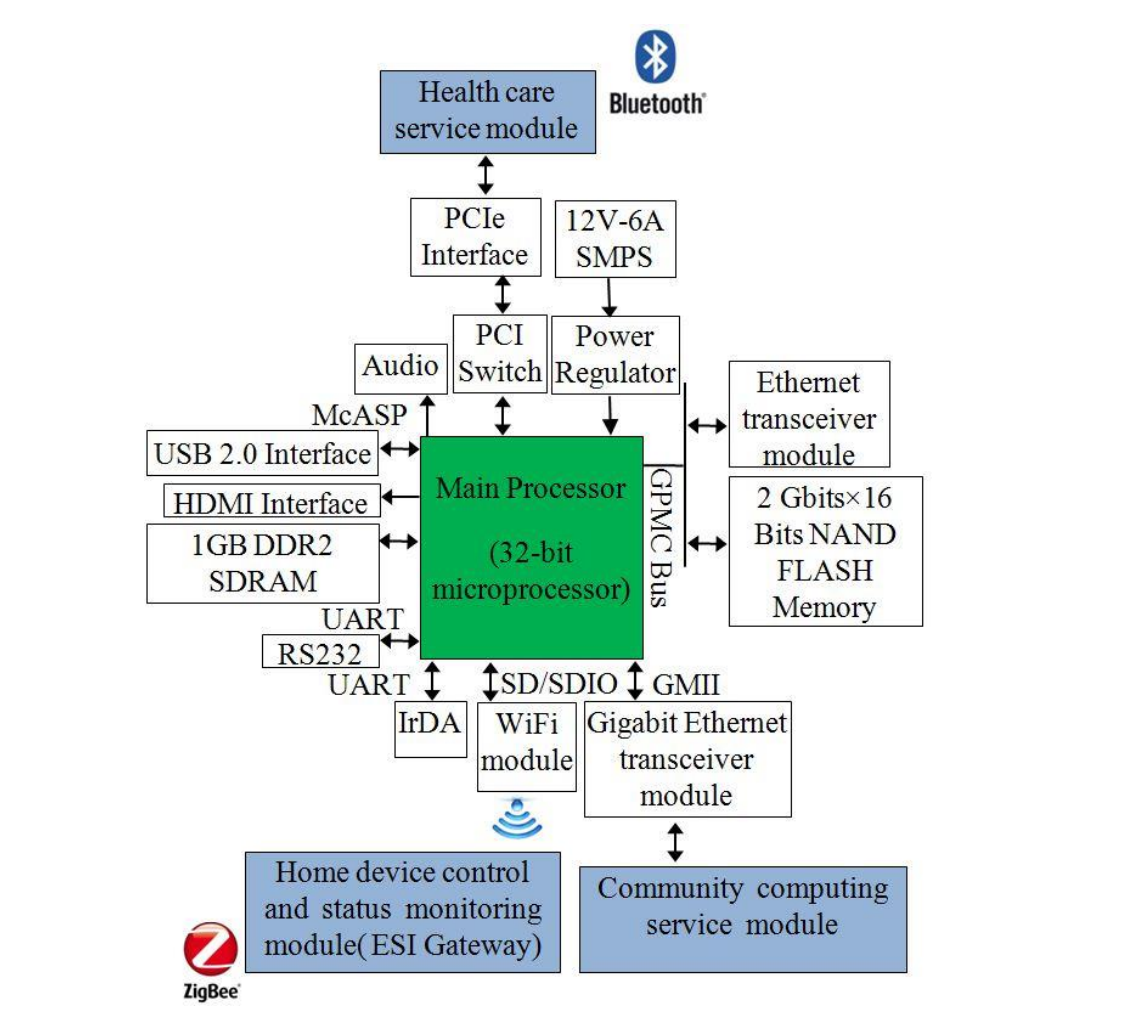

\subsection{The Power Section}

Figure 2. Block Diagram of a Modular Type Home Service Platform

The power section composes of power regulator and SMPS. This power regulator uses power management IC to divides the voltage according to needs of different circuit blocks (PLL, SATA, PCIe, DDR memory). Figure 3 shows the front panel of the developed home service platform and Figure 4 shows different service module interface with home service platform. 


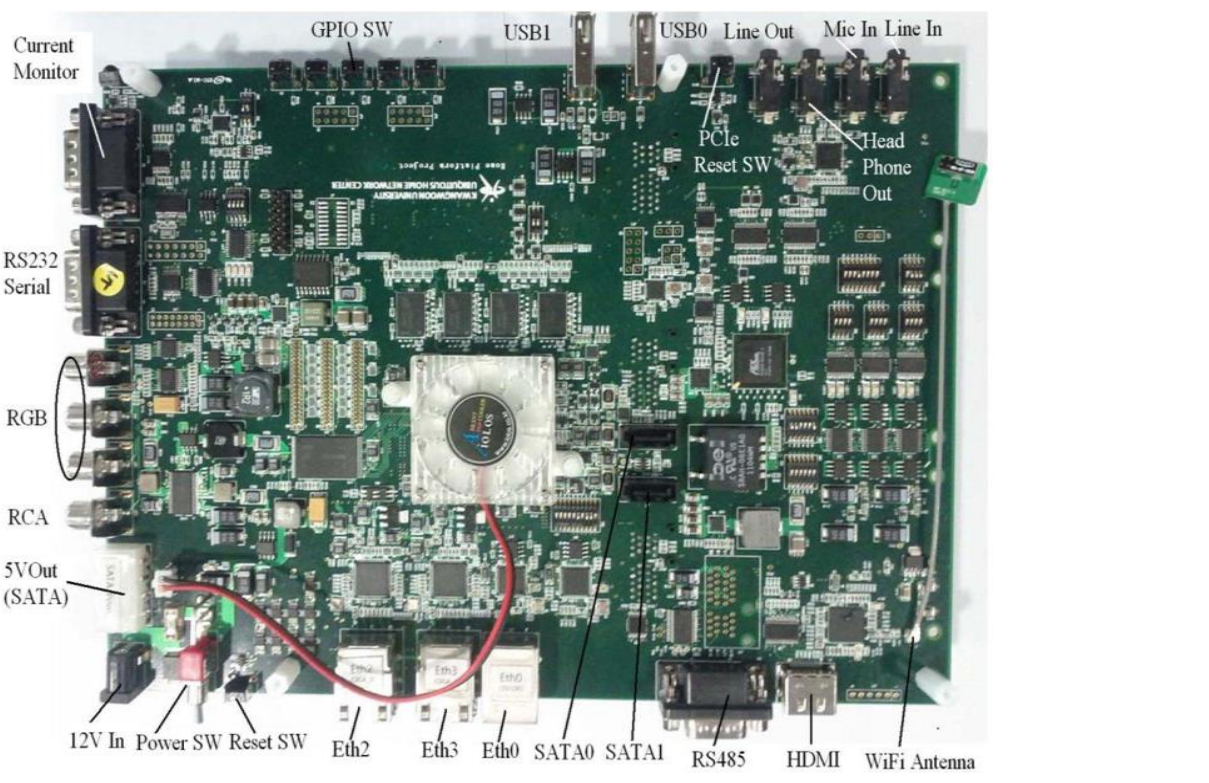

Figure 3. Front Panel of Modular Type Home Service Platform H/W System

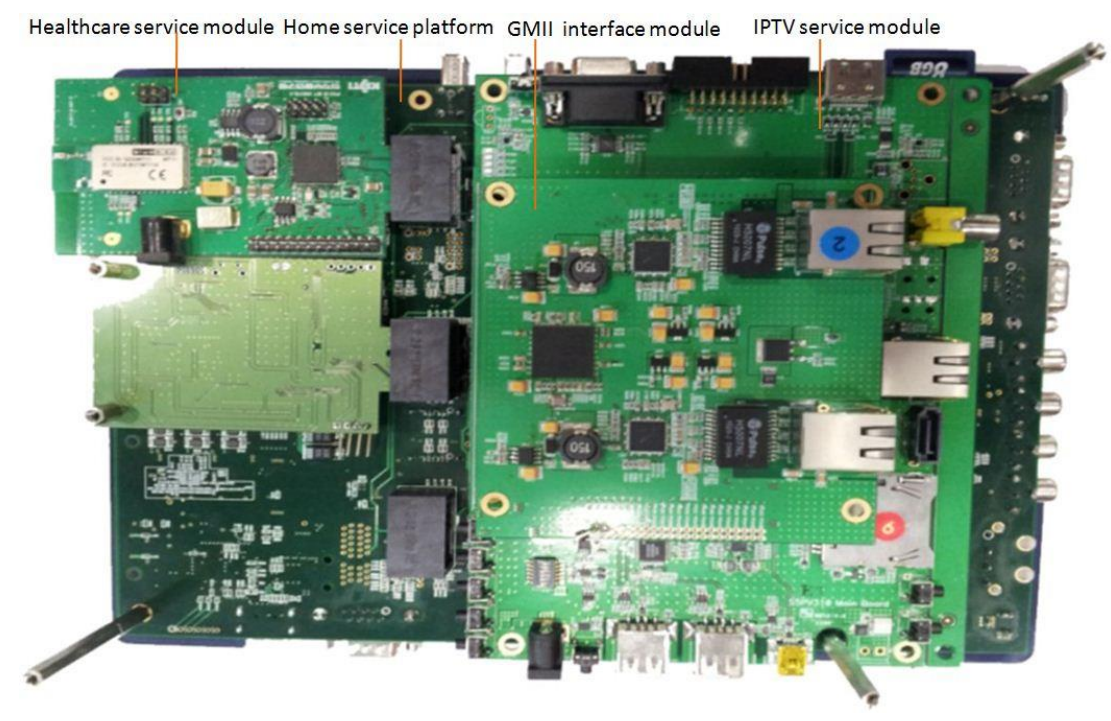

Figure 4, Different Service Module Interface with Home Service Platform

The operating system is one of the most important parts of the whole system. It is in charge of maraging all available resources and distributing them among different tasks efficiently. We are using Real-Time OS for the developed home service platform. The software architecture consists of three layers: the embedded OS kernel, application framework, and platform application (as shown in Figure 5). The embedded OS kernel is the basic layer. It provides basic system functionality like process and memory management and security. It handles network interface and device drivers for interface to peripheral hardware. It acts as an abstraction layer between the hardware and other software layers. The application framework provides many higher-level services to applications in form of classes. The application is the top layer of the architecture. It presents the features and capabilities of the system. 


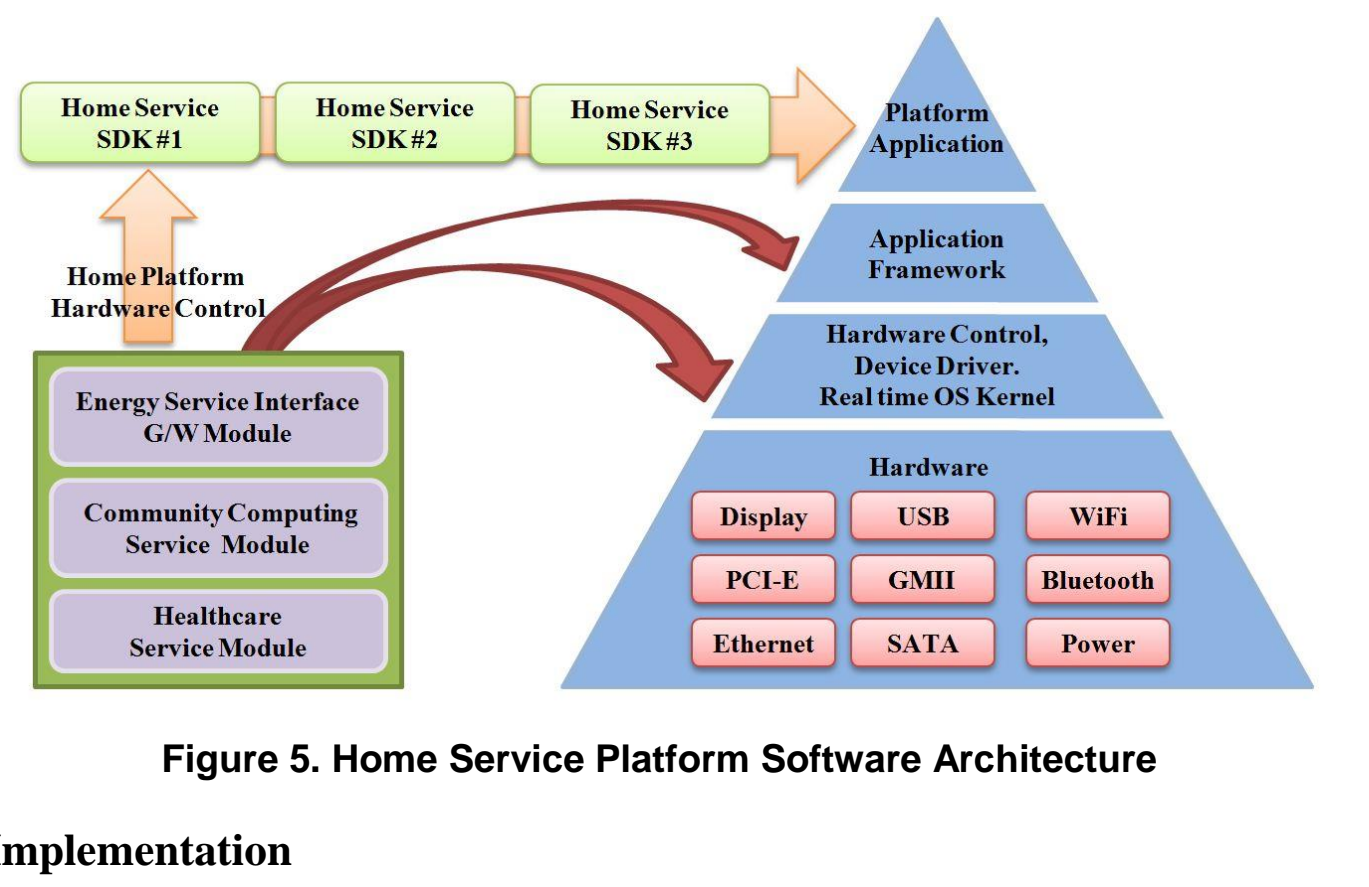

The developed home service platform was inplemented for three services: Home device control and status monitoring service, health care service and community computing service. Different types of modular hardware were designed and deyeloped for the three services. For the proper functioning of hardware, embedded operating system based driver software and application software was developed

\subsection{Home Device Control and Status Monitoring Management Services}

The home device contro and status monitoring management service controls and monitors the home devices (horne appliances). The ESI (Energy Service Interface) gateway uses Zigbee, a low-power and low-cost wireless personal area network standard (WPAN) based on IEEE 802.15.4 to Configure home the appliances network [9]. The Wi-Fi module is used for communication between the RS gateway and the home service platform module. The platform App was developed for use with this service. The energy management service gathers information and the controlled energy of different home appliances in use. The ESI gateway collects information on energy for the devices and plays the role of a gateway. If different communication methods are used for information collection then it will be extremely difficult because of the different possible processing methods. So, common standards and communication protocols are required. One standard is the smart energy profile. The smart energy profile based data transfer is designed to support smart appliances and provide a common communication environment. The home energy App works with the background service management module and the User Interface (UI) module. The home energy program App communicates with the Energy Service Interface gateway module (ESI) through either a wired LAN or wireless LAN (WiFi) and uses Zigbee to communicate with the home devices, or smart plugs as shown in Figure 6. The ESI acts as a bridge between the home service platform and home appliances. For the smart appliances function, the virtual control board is used with a Zigbee interface. The UI module displays simulation and monitoring data. 


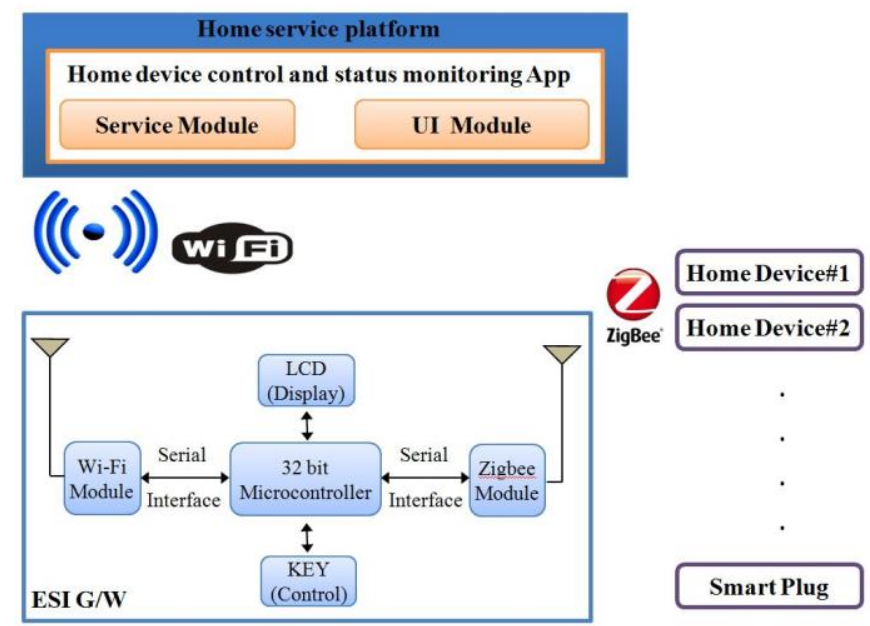

\section{Figure 6. Block Diagram of Home Device Control and Status Monitoring System}

The heart of the ESI gateway is a 32-bit microcontroller. The Zigbee and embedded Wi-Fi module's are serially interfaced with the microcontrollet. ESI gateway uses TCP/Server and end device uses TCP/Client protocol for communication. Following algorithms are used for energy monitoring and control bi-directional data llow.

1. Node list request/ response

Home platform App (PC appl.) sets up session and sends node list request to ESI gateway. Then the gateway retrieves the list of registered devices up to 0-9 node numbers and repeats the request. Algorithm is shownin Figure 7(a)

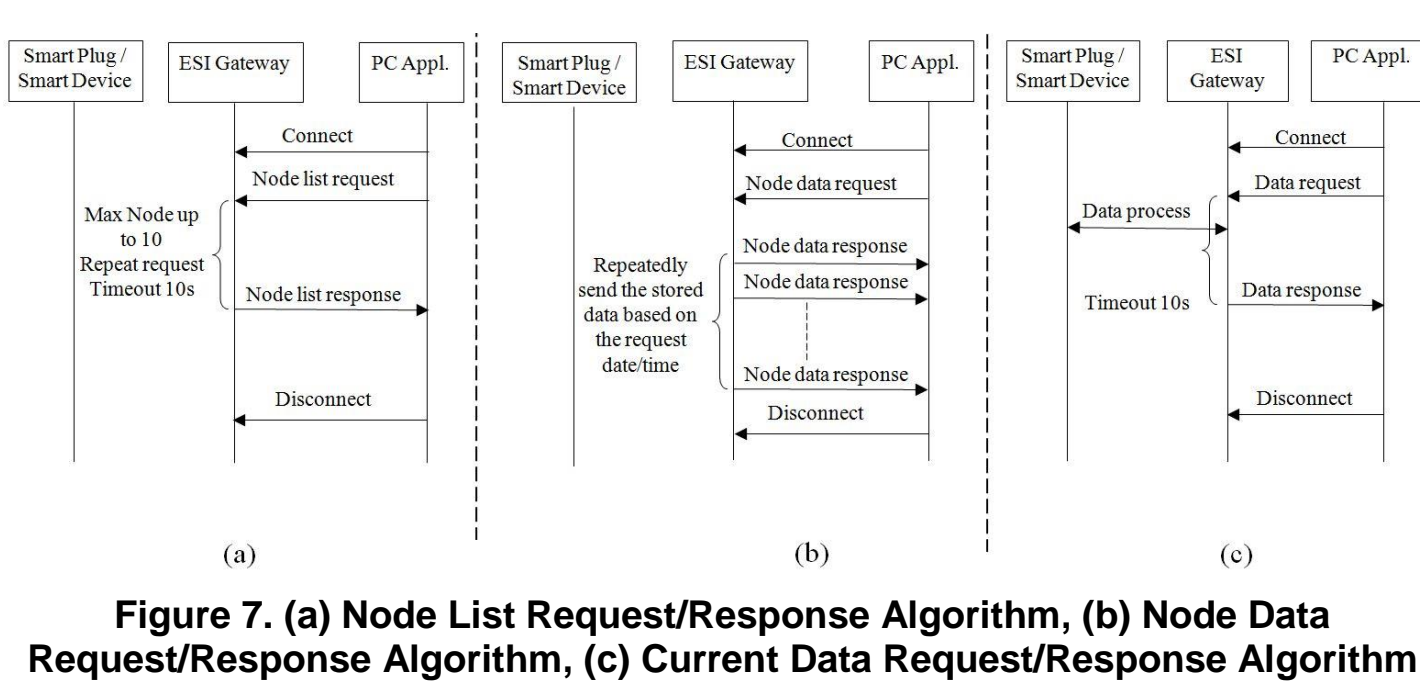

2. Node Data request/ response

Home platform App (PC appl.) sets up session and sends node data request to ESI gateway. ESI repeatedly sends stored data based on the date/time information. Date/time information consists of 6 byte to represent year, month, day, hour, minute 
and second. Year is based on 2000, 12 means year 2012. Algorithm is shown in Figure $7(b)$.

\section{Current Data request/ response}

Home platform App (PC appl.) sets up session and sends current data request to ESI gateway. ESI received data request, registered device data and respond to requests. Data request can be processed only one device at a time. If there is no response after 10 seconds of data request, then the request is deemed to have failed. Algorithm is shown in Figure 7(c).

\subsection{Health Care Service}

The health care service module receives the user's biometric information, stores it and then analyzes it. The health care service software is developed in the form of a platform App. This service module was authenticated by Continua Health Alliance (CHA). The Continua Health Alliance certified device is used to collect biometric information from the user's body. Bluetooth is used for communication between the health care service module and the end system. The health care service module software can process and display BP (Blood Pressure), BG (Blood Glucose), body mass and body weight information as shown in Figure 8.
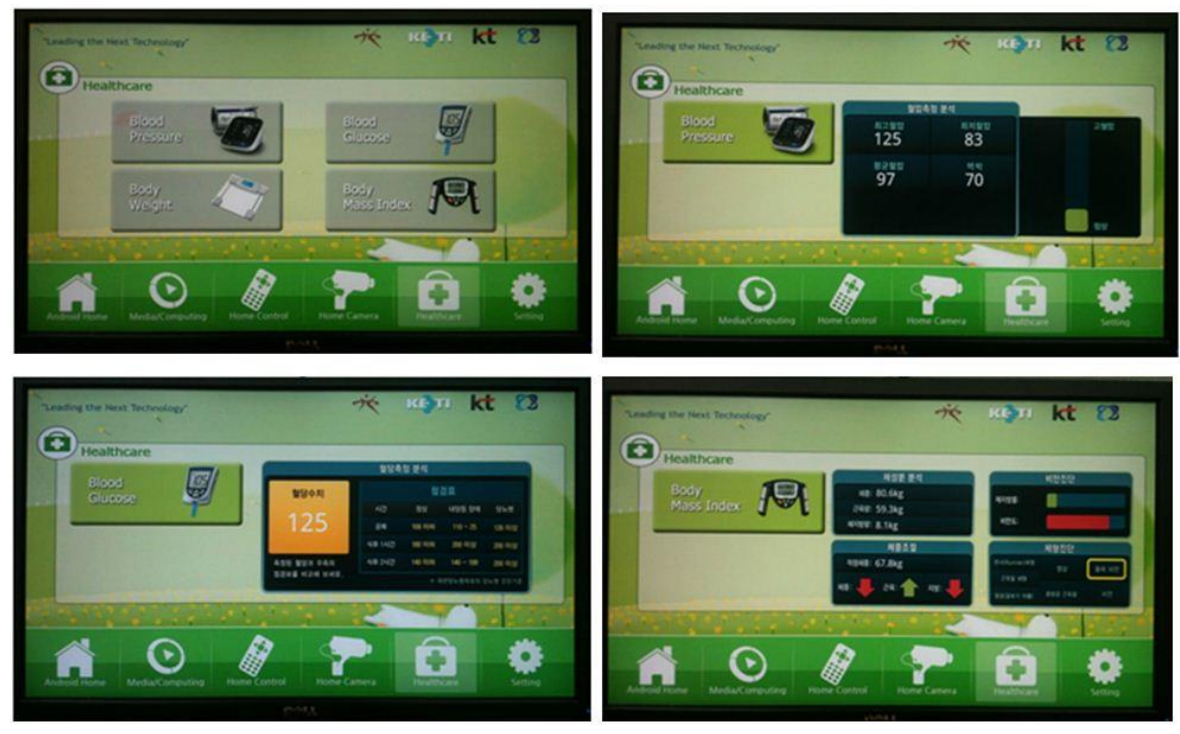

Figure 8. Health Care Service UI

The home health care service module supports biometric information acquisition based on the standards of CHA. CHA products use the IEEE11073-20601a Personal Health Data (PHD) protocol for communication between an agent and manager. The IEEE 11073 standard defines parts of a system to exchange vital sign and sensor data between different medical devices and the applications analyzing this data, as well as to operate these devices through a remote control. The scope is to establish common software architecture and communication between agents, these agents can then provide data that can be used either by medical measurement devices, sensor systems or managers, which analyze incoming data, without focusing on the transportation layer. Different standards offer plug-and-play and a functional as well as semantic interoperability between the sensor systems and aggregation systems. In order to work on the platform, the health care services use a PCI-Express interface to pass the 
healthcare data through the embedded operating system framework. The health care service S/W was designed in two sections. First, the platform based PCIe driver was developed. Secondly, the Health Device Profile (HDP) based on the platform App was developed for biometric information data collection, analysis and processing.

\subsection{Community Computing Service}

IPTV service module provides services based on community computing service. It uses GMII to interface with the home platform. IPTV module acts as a service agent for community computing service. Community network provides services to user inside and outside of home. User can enjoy service beyond the physical limits anytime, anywhere, any device. Home Everywhere Service (HES) in home network provides service by UPnP devices. Home network should be support UPnP internet gateway device to guaranty access from outside of home and home network proposes private network environmen yra home platform. Community computing network consists of HESC (Home Everywhere Service Client), HESA (Home Everywhere Service Agent), HESS (Home Everywhere Service Server) and IGD (Internet Gateway Device). Figure 9 shows HES conceptual diagram. HESC is a mobile terminal contained mobile devices such as mart phone, pad, etc. HESS store information of HESA location. IGD provides port forvarding on UPhP devices of home gateway.

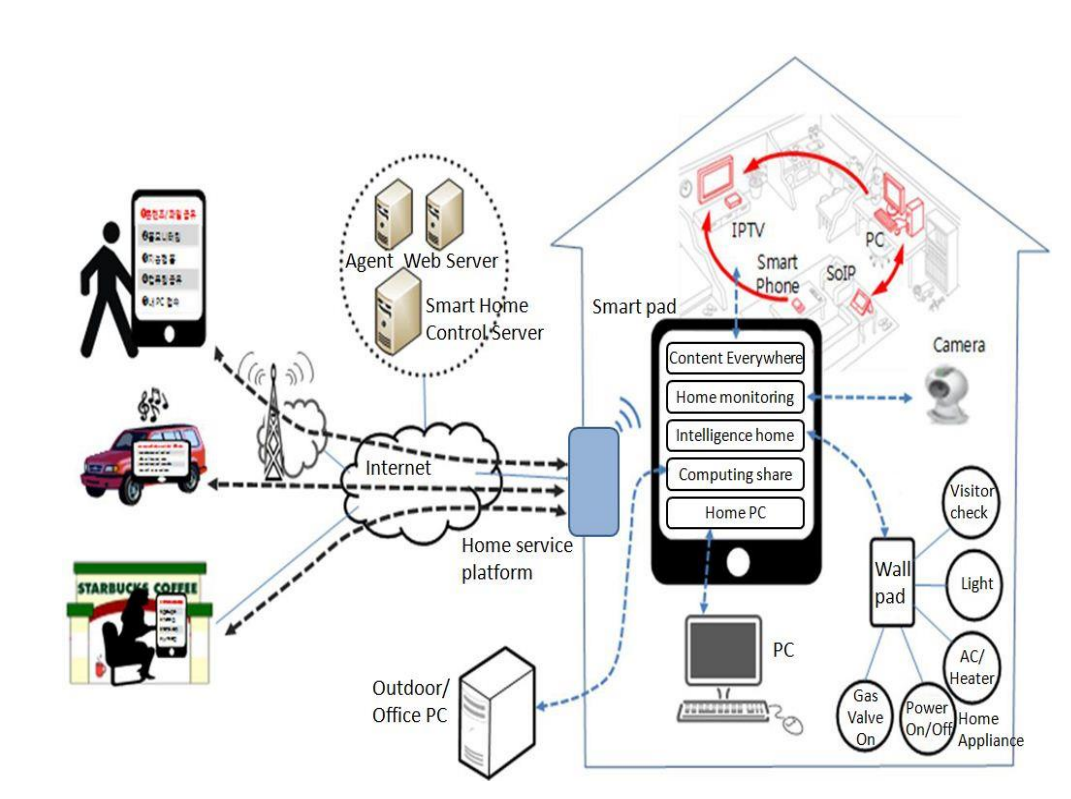

\section{Figure 9. Home Everywhere Service (HES) Conceptual Diagram}

Use (HESC) connects to HESA, and can get all device information within home. HESA gathers information of UPnP devices within home, and relay the information to the outside HESC on mobile device. After HESC receiving the device information, user can select a device among them, HESC request HESA for port forwarding to connect that device within home network. Figure 10 shows HES algorithm. In addition, HESC can register several HESA. So user can access to own home, own office and other's home if owner admit access. HESC communicate with HESA by SOAP message. 


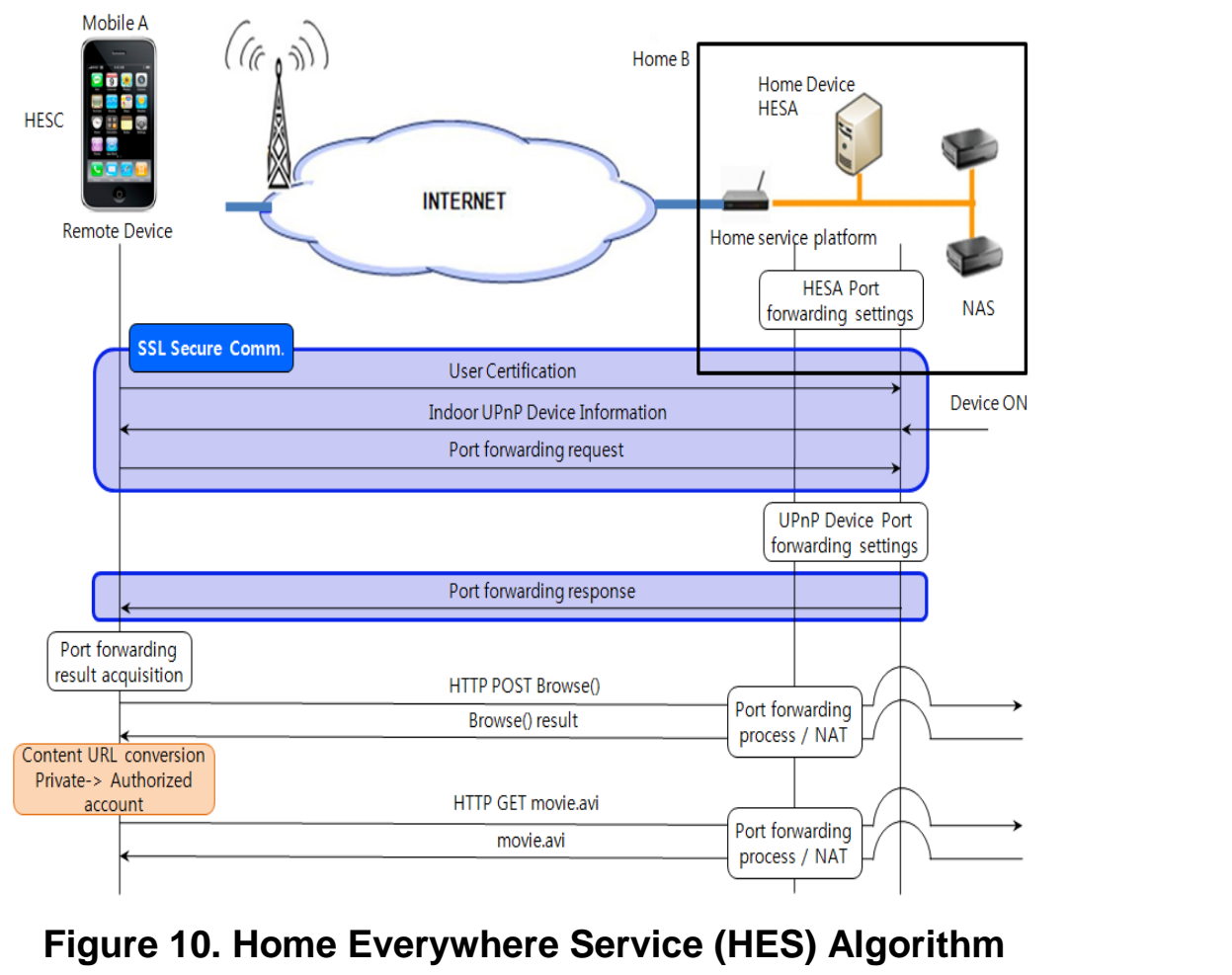

HESA platform App includes HESA and Renderer manager. It uses Community Computing File Server (CCFS). HESA manager uses HESA lib(.so) through platform interface and execute the request comes from UI. Through platform interface Renderer manager loaded renderer lib(.so) to interact with UI.

IP-TV software plays digital television media stream in MPEG-2 TS. For real time OS MPEG-2 stream player is used to play IP-TV stream. Stream player demultiplexed TS which is delivered by RTP LP-TV software composed of core control, input, demuxer, decoder and rendere modules. Decoder and renderer are composed of audio decoder, video decoder, audio out and video modules. Input module gets the media data through socket interface and the data goes thorough Demuxer module to extract the audio and video stream. Decoder is used to decode the audio and video data. After decoding, the audio data is send to audio output and video data to video output. Core control modules controls all the modules. IP-TV software architecture consists of three layers: Application layer, application framework, real time OS kernel (as shown in Figure 11). Real time IP-TV software was developed on top of the application framework using API. Application layer takes the role of user interface and screen layer configuration. User is interfaces with this layer. Figure 12 shows the UI screen of IP-TV module. Real time OS kernel is-modified for IP-TV H/W driver. Main function of real time IP-TV are channel scan, channel list prepare, channel watching, favorite channel management, EPG (Electronic Program Guide) data creating, reserved recording, real time channel transferring, UPnP device search, webpage remote control. 


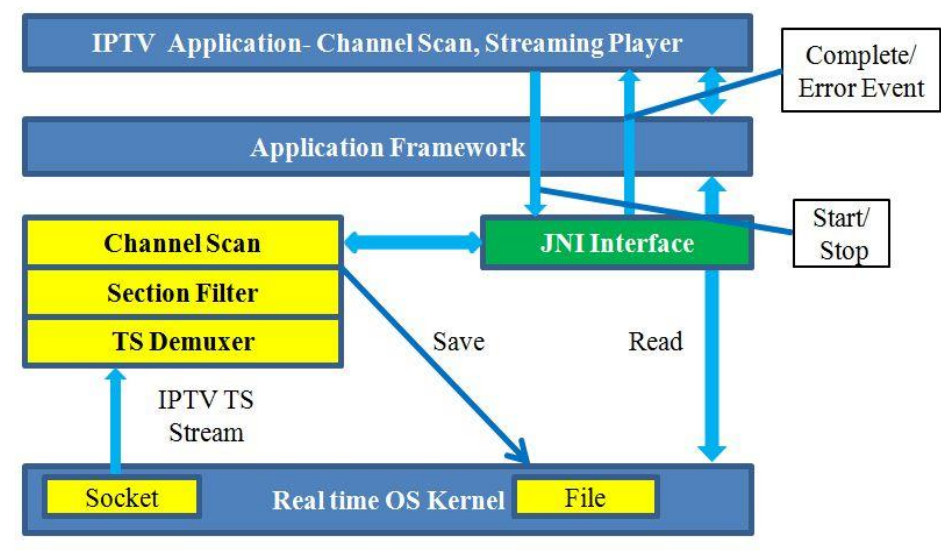

H/W

Figure 11. IPTV Software Architecture

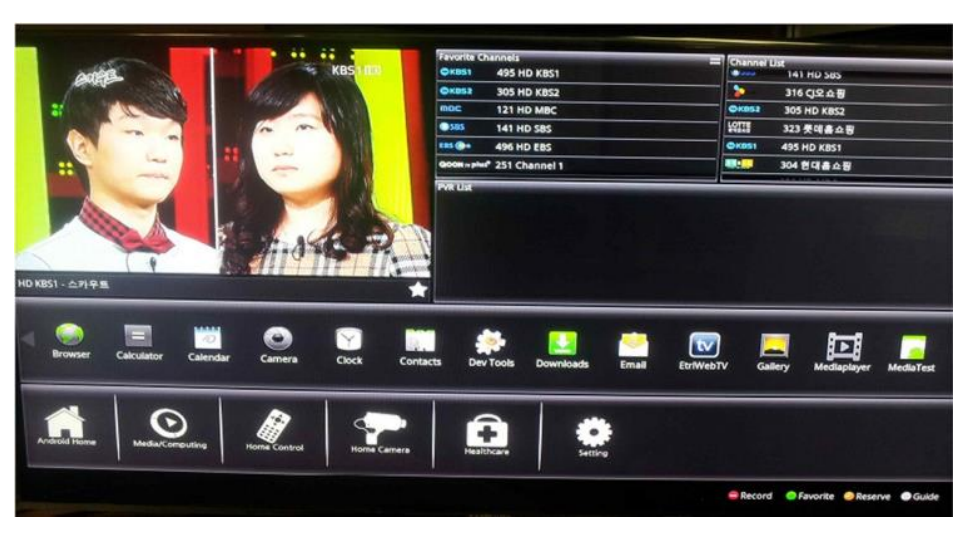

Figure 12. IPTV Capture Screen

\section{Conclusion}

We developed an ideal platform for convergence services in home network. This platform uses a low-cost, power efficient, high performance microprocessor with various types of interface facility. This blatform is of a modular type providing it with flexibility, simplicity and expandability. This modular type platform with a real time embedded operating system implements 3 services, home device control and status monitoring management service, health care services, as well as community computing network service. It is also possible to add other services. This module provides a seamless communication platform for different services. It is the first step towards making a context aware system for smart home environment.

\section{Acknowledgements}

This work was supported by the Industrial Strategic Technology Development Program funded by the Ministry of Trade, Industry \& Energy (MI, Korea) and Research Grant of Kwangwoon University in 2013. 


\section{References}

[1] N. Kushiro, S. Suzuki, M. Nakata, H. Takahara and M. Inoue, "Integrated residential gateway controller for home energy management system," IEEE Transaction on Consumer Electronics, vol. 49, no. 3, (2003), pp. 629-636.

[2] B. Rose, "Home networks: a standards perspective," IEEE Communication Magazine, vol. 39, no. 12, (2001), pp. $78-85$.

[3] A. Tsutsui, "Latest trends in home networking technologies," IEICE Transaction on Communication, vol. E91-B, no. 8, (2008), pp. 2470-2476.

[4] S. Guangming, W. Zhigang, Z. Weijuan and S. Aiguo, "A multi-interface gateway architecture for home automation networks," IEEE Transaction on Consumer Electronics, vol. 54, (2008), pp. 1110-1113.

[5] L. Jiang, D- Y. Liu and B. Yang, "Smart home research," Proceedings of the Third IEEE Conference on Machine Learning and Cybernetics , (2004) August 26-29; Shanghai, China.

[6] F. paganelli and D. Giuli, "An ontology-based system for context-aware and configurable services to support home-based continuous care," IEEE Transaction on Information Technology in Biomedicine, vol. 15, no. 2, (2011), pp. 324-333.

[7] F. Birlik, O. Gurbuz, and O. Ercetin, "IPTV home networking via 802.11 wireless mesh networks: an implementation experience," IEEE Transaction on Consumer Electronics, vol. 55, no. 3, (2009), pp. 11921199.

[8] A. Kusiak and C- C. Huang, "Development of modular products, NEEE transaction on Components, Packaging, and Manufacturing Technology-Part A, vol. 19, no. 4 (1996), pp. 523-538.

[9] S. Park, E. J. Lee, J. H. Ryu, S- S. Joo, and H. S. Kim "Distributed borrowing addressing scheme for ZigBee/IEEE 802.15.4 wireless sensor networks," ETRI Journal, vol. 31, no. 5, (2009), pp. 525-533.

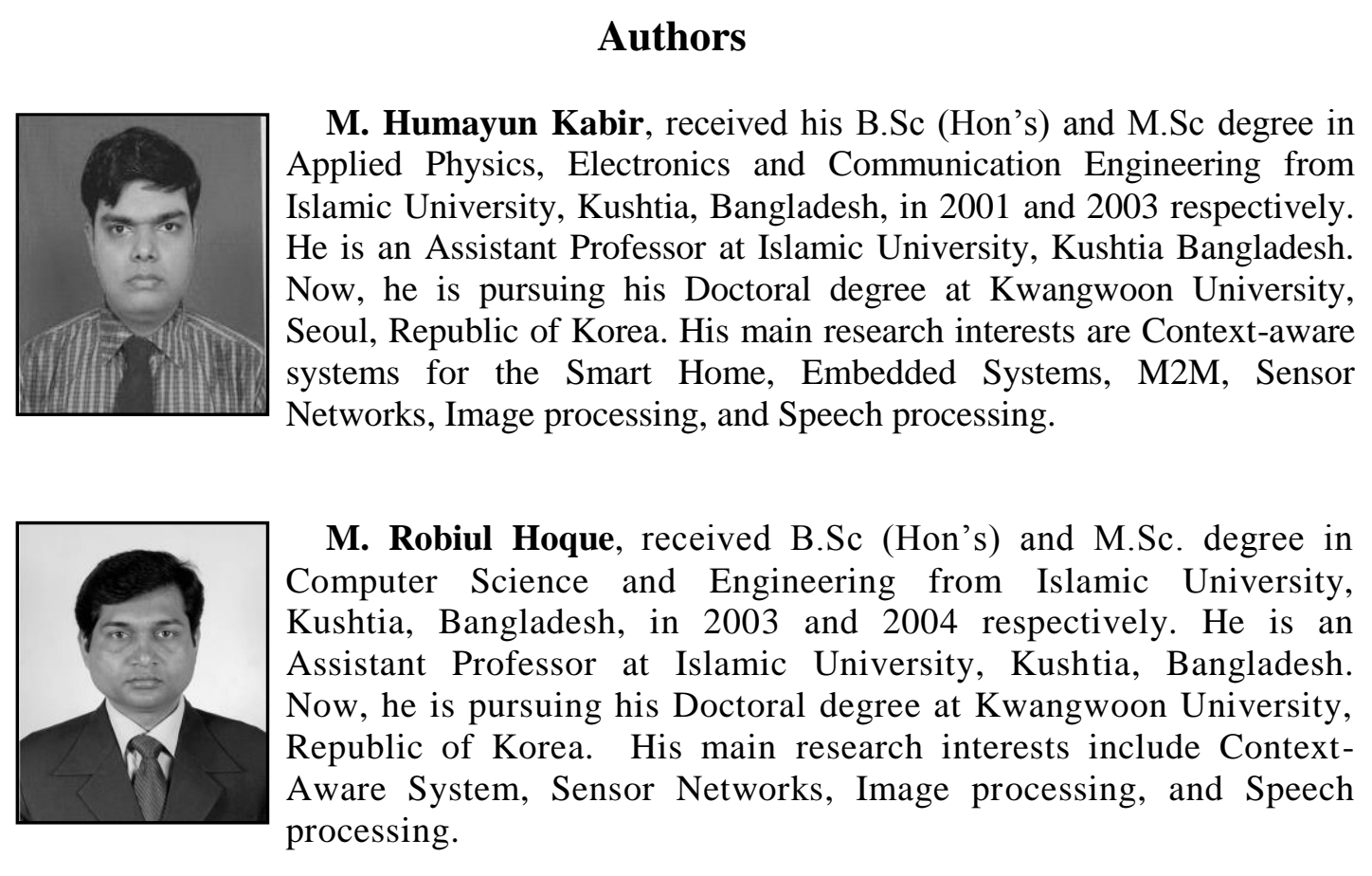

Kangji Lee, received his Bachelor of Engineering degree in Computer

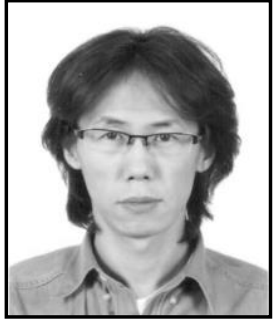
Engineering from Hankuk University of Foreign Study, Seoul, Republic of Korea in 2001. He is working as Senior Research Engineer at MIware Inc. Gyeonggi-do, Republic of Korea. His main research interest is multimedia. 


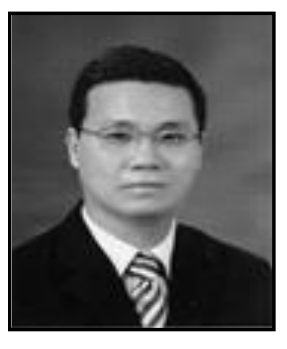

Chang-Won Park, received his Bachelor degree in Electronic Engineering from Chung Ang University, Seoul, Republic of Korea in 1986 and Master's degree in Information and Communication Technology from Kwangwoon University, Seoul, Republic of Korea in 2002. He completed his Ph.D. in Computer Engineering from Sungkyunkwan University, Seoul, Republic of Korea in 2006. He has been working on researches in Department of ICT/Medical IT Convergence Research Center, Korea Electronics Technology Institute since 1993, and currently assuming the director of Industry of Medicare IT. His main research interests are Wellness Care, Medicare IT, PMS, and Medical Network.

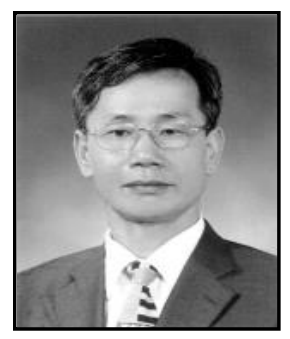

Sung-Hyun Yang, received his B.S. and M.S. degree in Electrical Engineering from Kwangwoon University, Seoul, Republic of Korea, in 1983 and 1987 respectively. He completed his Ph.D. from Kwangwoon University in 1993. He is a Professor in Electronc Engineering at Kwangwoon University, Seoul, Republie of Korea He is a Director of the Ubiquitous Home Network Center, Kwangwoon University. He was a Research Scientist at Boston University from 1996 to 1998. He was Chairman of the Home Network Market Activation Section, Korean Association for Smart Home from 2007 to 2008. His main research interests are Digital logic, Embedded Systems, M2M, Next Generation Ubiquitous Home Networks, añd Context-aware systems.

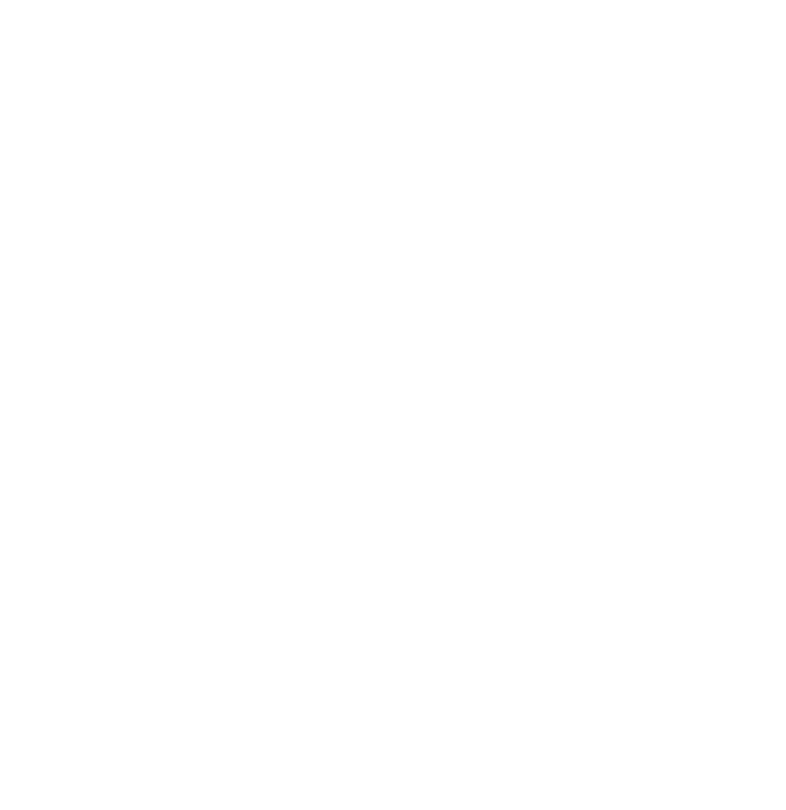


International Journal of Smart Home

Vol.8, No.4 (2014)

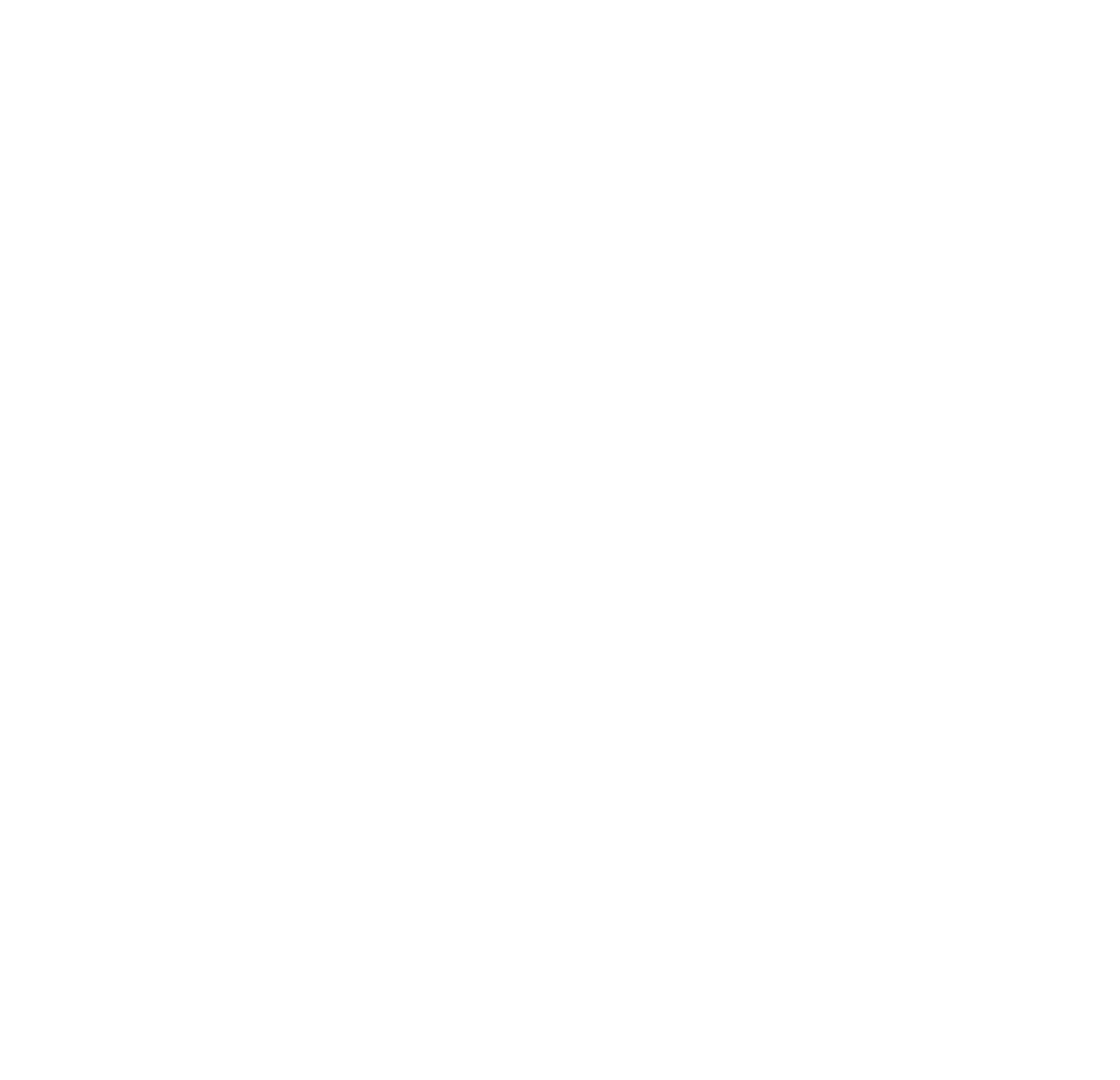

